\title{
MRF Based LSB Steganalysis: A New Measure of Steganography Capacity
}

\author{
Debasis Mazumdar ${ }^{1}$, Apurba Das ${ }^{1}$, and Sankar K. Pal ${ }^{2}$ \\ 1 CDAC, Kolkata, Salt Lake Electronics Complex, Kolkata, India \\ \{debasis.mazumdar, apurba.das\}@cdackolkata.in \\ 2 Indian Statistical Institute, Kolkata, India \\ sankar@isical.ac.in
}

\begin{abstract}
A new steganalysis algorithm is described based on the MRF model of image LSB plane. In this framework the limitation of the Cachin's definition of the steganography capacity is quantified and a new measure is proposed.
\end{abstract}

Keywords: Steganography, Cover object, Stego object, Ising model, partition function, $\epsilon$-security.

\section{Introduction}

The classical definition of steganography is statistical and not perceptual [1]. Determining steganography capacity is an important research topic. As per Cachin's 2 definition a steganography scheme is said to be $\epsilon$-secure if the KullbackLeibler(K-L) divergence between the stego and the cover object is at most $\epsilon$. However, in this model the statistical properties of the cover image has not been considered as a parameter. In the present work an attempt has been made to quantify the limitations of the Cachin's formula experimentally and a new measure of steganography capacity which is more relevant to the real life data is proposed.

We have developed a new steganalysis algorithm in which the LSB plane is modeled as a 2D Ising lattice, and Gibbs Markov statistical distribution is considered to be the a-priory distribution over the lattice. Using this model we have described the limitation of Cachin's $\epsilon$-security in case of natural images. In the framework of Ising model the local randomness in the LSB plane is studied and its influence on the steganography capacity is computed. Based on these a new measure of steganography capacity is then derived. It is also shown that our theoretical model is coherent and in perfect agreement with the real life situations.

\section{Statistical Structure of the LSB Plane}

The statistical structure of the LSB plane is apparently very random, possessing no correlations, even among the neighbors. The heterogeneity or the disorderliness present in the LSB plane makes the process of detecting tampered bits more and more complex. 
In Table-1 we represent some natural images, distribution of 1 and 0 in their LSB plane (white and black dots correspond to LSB value 1 and 0 respectively) and coefficient of kurtosis $\left(\alpha_{4}\right)$ of the distribution. It is clearly observed that, in some images, namely the first two, the coefficient of kurtosis is less than 3 and their LSB plane are highly random. In the other cases, the coefficient of kurtosis are more than 3 and long sequences of homogeneous LSBs are present. The images for which $\alpha_{4}<3$, detection of tampered bit is complicated. In the other set of images where $\alpha_{4}>3$, the detection is easier. The sharpness of the distribution in the latter case helps us in detecting the outliers easily. In order to develop a coherent steganalysis algorithm based on this statistical property of the LSB plane, we describe it as a 2D ising lattice having Gibbs-Markov probability distribution.

Table 1. Coefficient of kurtosis of the distribution of ' 1 ' and ' 0 ' in the LSB plane for different images

\begin{tabular}{|c|c|c|c|c|c|c|c|c|}
\hline \multicolumn{9}{|c|}{ Table1 } \\
\hline Image & ISB & $\alpha=\frac{(x-g)^{\prime}}{\sigma^{4}}$ & Image & ISB & $a_{4}-\frac{B(X-X)}{\sigma^{4}}$ & Image & LSB & $\alpha_{1}-\frac{Y(X-Y)^{\prime}}{\sigma^{4}}$ \\
\hline & & 1.0007 & 50 & & 5.3354 & & & 11.294 \\
\hline & 80 & 1.0848 & & & 9.6977 & 6 & & 14.255 \\
\hline
\end{tabular}

\section{Ising Model of the LSB Plane}

In our model, the LSB plane is represented as a 2D Ising lattice having the pixel values \pm 1 . LSB based steganographic techniques either change the pixel values by \pm 1 or leave them unchanged. In this Ising lattice, let us introduce the notation $\eta_{i j}$ representing the neighborhood of the $(i, j)^{t h}$ pixel. If $\eta_{i j}$ is perfectly homogeneous and isotropic, the entire lattice will be perfectly selfsimilar with respect to a basic building block, otherwise the heterogeneity or disorderliness sets in. We will show in the later part of the present paper that the fluctuation in the configuration of $\eta_{i j}$ plays a significant role in determining the steganography capacity of the cover image. In order to introduce further geometrical notion into the model let us define $\Re_{i j}$ as the set of cliques associated with the neighborhood $\eta_{i j}$. The examples of neighborhood system and their associated cliques are discussed in many books and technical papers 3. Let, $(\xi, \tau, \rho)$ define a Markov Random Field (MRF) over this Ising lattice, where $\xi$ defines the set of all possible configurations, called the sample space, $\tau$ is the collection of subsets of $\xi$ and $\rho$ is the Gibbs distribution on this lattice, defined as:

$$
\rho(x)=\frac{1}{Z} e^{-u(x)}
$$


where $\mathrm{u}(\mathrm{x})=\sum_{\forall(i, j)} u_{C}\left(x_{C}\right)$. Here $\mathrm{u}(\mathrm{x})$ is the potential function, $u_{C}\left(x_{C}\right)$ is the energy associated with the cliques $\mathrm{C} \in \Re_{i j}$, and $\mathrm{Z}=\sum_{\forall x} e^{-u(x)}$ is the normalizing constant, popularly known as the partition function. In our present work we have utilized a particular class of MRF with pair-wise interaction to model the LSB plane. This class of model has been extensively used for restoration of degraded binary images [4]. These applications have a close resemblance to our present problem. The joint density function (Eq.11), in our model considers the first order neighborhood system where in each pixel interacts with its immediate neighbors as shown in the Fig. 1. We further assume that, the clique interaction energy follows the Hebbian rule [3]. Let, $\beta_{h}$ and $\beta_{v}$ be the coupling intensities in the horizontal and vertical direction respectively and $(i, j),(i \prime, j \prime)$ is a clique. The generalized expression of the interaction potential is:

$$
u\left(x_{i j}\right)=\beta_{h} \sum x_{i j} x_{i \prime j^{\prime}}+\beta_{v} \sum x_{i j} x_{i \prime j \prime}
$$

We describe in the next section the algorithm to estimate the values of $\beta_{h}$ and $\beta_{v}$ for any image.

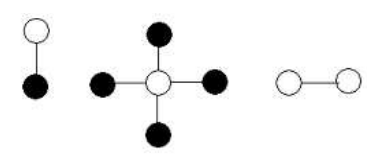

Fig. 1. Horizontal and vertical cliques

\section{Estimation of the MRF Parameters and Detection of Tampered Bits}

The problem of parameter estimation is an aspect of the research related to the modeling of any image using MRF. The principal cause of criticality lies in the computation of the partition function Z. In the present work EM(Expectation Maximization) based algorithm proposed by Besag et. al [5], is used to estimate the MRF parameters. Once $\beta_{h}$ and $\beta_{v}$ is estimated, the probability of occurrence $\rho(x)$ of each pixel is calculated using Eq. 1. If $\rho(x)$ is found to be significantly small, the corresponding pixel is considered as an outlier with respect to its neighborhood and declared as a tampered bit. In a complete image if the number of tampered bits crosses a threshold, then the image is declared as a stego image. In the following it will be shown that, the local randomness of the bit pattern around a pixel of the LSB plane is an important parameter in determining the accuracy of computation of $\rho(x)$. If for some random bit pattern gross inaccuracy occurs in the computation of $\rho(x)$ then its detection as stego/non-stego will be grossly inaccurate. We can say that such bit pattern offers more security to be considered as a secured cover image and the steganography capacity can be considered as a function of the $\%$ error of detecting tampered and untampered 
bits. In the next section we describe the influence of local randomness in the LSB plane on the estimation of tampered/ un-tampered bits. Based on this result a new measure of steganography capacity is also proposed.

\section{Run Statistics of the LSB Plane and a New Measure of Steganography Capacity}

The new steganalysis algorithm as described above, is tested over a large number of natural images. For space restriction a few results are presented here. In Fig. 2 the $\%$ of erroneously detected tampered and un-tampered bits is plotted as a function of the co-efficient of kurtosis $\left(\alpha_{4}\right)$, of the LSB plane. It is observed that for the images with high co-efficient of kurtosis $\alpha_{4}$, detection of tampered/ un-tampered bits is less erroneous. It is also to be noted that, for small values of $\alpha_{4}$, the detection accuracy is very poor and nearly the same for both the stego and non-stego images. As $\alpha_{4}$ increases, the discernibility between the stego and non-stego images increases. To bring further clarity into this explanation we use the run statistics of images. We assume that any image can be considered as a set of rows (or columns). In each row, let there be $\mathrm{N}$ partition points (rank) which separate the sequences of homogeneous pixels (Fig. 3a. If in a row, there are $N_{A}$ number of +1 pixels and $N_{B}$ number of -1 pixels, the z-score of the Run-statistics is defined as [6]:

$$
z=\frac{N-\mu_{v}}{\sigma_{v}^{2}}
$$

where,

$$
\mu_{v}=\frac{2 N_{A} N_{B}}{N_{A}+N_{B}}+1 \quad \text { and } \quad \sigma_{v}=\frac{2 N_{A} N_{B}\left(2 N_{A} N_{B}-N_{A}-N_{B}\right)}{\left(N_{A}+N_{B}\right)^{2}\left(N_{A}+N_{B}-1\right)}
$$

Let, the null hypothesis $H_{0}$ be that 'the row is random' and $H_{1}$ be the alternative hypothesis that 'the row is homogeneous'. For a two tailed test at the significance level 0.05 , a row will be considered as random when $-1.96 \leq z \leq 1.96$ otherwise it will be considered that the row has long run length. In Fig. 3 b the percentage

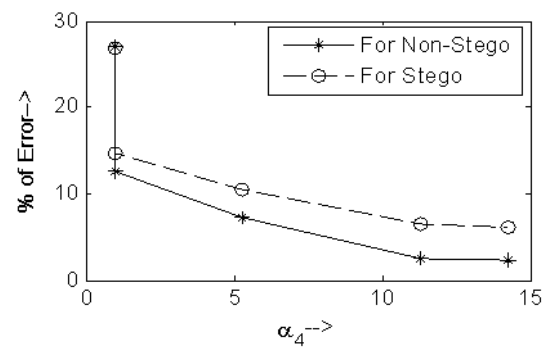

Fig. 2. Change in reconstruction error with coefficient of kurtosis 


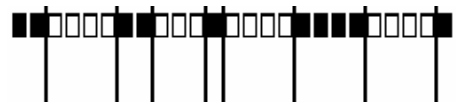

(a)

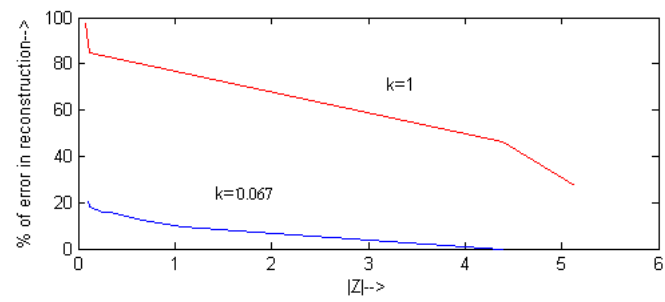

(b)

Fig. 3. (a) Partition points(rank) separating the sequences of homogeneous pixels, and (b) Change in reconstruction error(\%) with Z-score

error(e) in reconstruction is plotted as a function of the z-score. The curve is estimated numerically and a polynomial is obtained which fits into the curve closely.

$$
e=k\left(-15.386|Z|^{3}+145.275|Z|^{2}-359.339|Z|+125.5252\right)
$$

where $\mathrm{k}$ is a parameter which takes different positive non-zero values for different set of images. We now define the modified measure of steganography capacity as $\beta_{\aleph}$ where

$$
D\left(P_{S} \| P_{C}\right)+\frac{1}{k\left(-15.386|Z|^{3}+145.275|Z|^{2}-359.3393|Z|+125.525\right)}<\beta_{\aleph}
$$

$D\left(P_{S} \| P_{C}\right)$ represents the $\mathrm{K}-\mathrm{L}$ divergence between the cover and stego image as taken into consideration by Cachin in his definition of $\epsilon$-security.

\section{Experimental Results}

We compute the K-L divergence between a pair of stego and non-stego images using the formulae:

$$
D\left(P_{C} \| P_{S}\right)=\log \frac{Z_{S}}{Z_{C}}-E_{C}\left[u_{C}-u_{S}\right]
$$

where $Z_{S}$ and $Z_{C}$ are the partition functions of the stego and cover image respectively and $u_{S}$ and $u_{C}$ are the clique potentials in the stego and cover image. $E_{C}[]$ represents the expectation of any random variable with respect to the distribution of the non-stego images. The K-L divergence for a number of natural images are computed. The images are chosen cautiously such that for each of them the K-L divergence remain nearly the same for particular amount of packing density. Following Cachin's definition, it is expected that, the \% of error in bit reconstruction will remain same for all these images. In the Fig. 4 we see that the experimental curve is significantly deviated from the theoretical curve following Cachin's formulae. While the curve obtained using our formula is in close agreement with the experimental curve. 


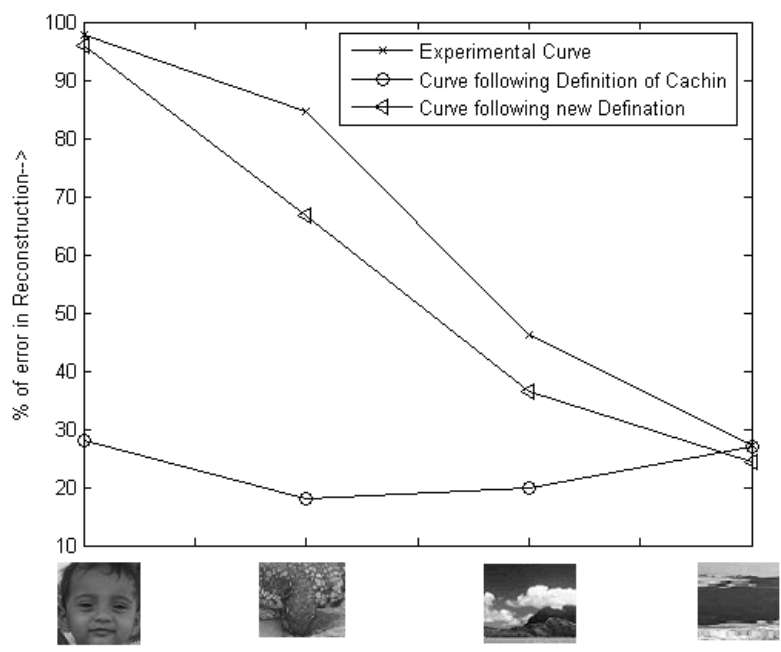

Fig. 4. Variation of the reconstruction error in different images. Experimental curve, curve obtained using Cachin's formula and the proposed measure of steganography capacity.

\section{References}

1. Mazumdar, D., Mitra, S., Dhali, S., Pal, S.K.: A chosen plaintext steganalysis of hide4pgp v 2.0. In: Pal, S.K., Bandyopadhyay, S., Biswas, S. (eds.) PReMI 2005. LNCS, vol. 3776, pp. 459-464. Springer, Heidelberg (2005)

2. Cachin, C.: An information-theoretic model for steganography. Information and Computation 1, 41-56 (2004)

3. Geman, S., Geman, D.: Stochastic relaxation, gibbs distributions, and baysian restoration of images. IEEE Trans. Pattern Anal. and Mach. Intell. 16, 721-741 (1984)

4. Yamasaki, T., Shirazi, M.N., Noda, H.: Adaptive restoration of degraded binary mrf images using em method. IEEE Transaction on Information and Systems E76D, 259-268 (1993)

5. Besag, J.E.: Spetial interaction and the statistical analysis of lattice system. J. Roy. Statis. Soc. B, 36, 192-336 (1974)

6. Spiegel, M.R., Schiller, J., Srinivasan, R.A.: Theory and Problems of Probability and Statistics, 2nd edn. Tata McGraw-Hill Publishing Company Ltd. (1998) 